\title{
Estudo comparativo entre cinco diferentes tratamentos sobre as alterações clínicas e laboratoriais do rato diabético induzido pela aloxana ${ }^{1}$
}

\author{
Comparative study among five different treatments on the clinical and laboratory \\ changes of the alloxan-induced diabetic rats
}

\section{César Tadeu Spadella², Célia Sperandéo Macedo³ ${ }^{2}$ José Lúcio Martins Machado ${ }^{4}$, Silvana Artioli Schellini ${ }^{5}$, Carlos Roberto Padovanni ${ }^{6}$}

1. Trabalho realizado no Laboratório de Técnica Cirúrgica e Cirurgia Experimental da Faculdade de Medicina de Botucatu (FMB) - UNESP.

2. Prof. Adjunto da Disciplina de Gastroenterologia Cirúrgica, do Departamento de Cirurgia e Ortopedia, da FMB - UNESP.

3. Profa. Adjunta do Departamento de Pediatria da FMB - UNESP.

4. Prof.Dr. da Disciplina de Pediatria, do Departamento de Cirurgia e Ortopedia da FMB - UNESP.

5. Profa. Adjunta do Departamento de Oftalmologia e Otorrinolaringologia da FMB - UNESP.

6. Prof. Titular do Departamento de Bioestatística, do Instituto de Biociências de Botucatu - UNESP.

\section{RESUMO}

Objetivos: Este estudo visa a analisar os efeitos, a longo prazo, de cinco diferentes tratamentos sobre o controle metabólico de ratos diabéticos aloxânicos. Métodos: Foram analisados 7 grupos experimentais, com 50 ratos cada um, sendo: GN o grupo controle normal; GD o grupo controle diabético, sem tratamento; GI, GA e GIA os grupos tratados, respectivamente, com insulina, acarbose e associação insulina + acarbose; GTIL o grupo tratado com transplante de ilhotas de Langerhans; e o GTPD o grupo tratado com transplante pancreatoduodenal heterotópico. Parâmetros clínicos (peso, ingestão hídrica, ingestão alimentar e diurese) e laboratoriais (glicemia, glicose urinária e insulina plasmática) foram avaliados em todos os animais, no início do experimento, e após 1, 3, 6, 9 e 12 meses de seguimento. Resultados: À exceção do GN, mortalidade foi observada em todos os grupos experimentais no seguimento de 12 meses (GD=50\%; GI= 20\%; GA= 26\%; GIA=18\%; GTIL=4\%; GTPD=20\%). Em GD, GI, GA e GIA os óbitos ocorreram por distúrbios metabólicos ou hidroeletrolíticos e/ou pneumonia, diarréia e caquexia; em GTIL e GTPD todos os óbitos ocorreram por falhas técnicas no pós-operatório até 72h. Animais dos grupos GI, GAe GIA tiveram melhora significativa $(\mathrm{p}<0,05)$ de todos os parâmetros clínicos e laboratoriais observados em ratos diabéticos, sem diferença de efetividade entre os tratamentos. Porém, os resultados observados nestes grupos, biologicamente não foram comparáveis aos observados em GTIL e GTPD, onde observou-se correção completa, aos níveis normais, de todas as variáveis analisadas $(\mathrm{p}<0,01)$. Conclusões: Os tratamentos convencionais com insulina, acarbose e insulina + acarbose melhoraram o estado diabético grave dos ratos tratados, contudo, a eficácia dos tratamentos foi significativamente inferior à oferecida pelo GTIL e GTPD.

Descritores: Diabetes. Rato. Aloxana. Insulina. Acarbose. Transplante de Pâncreas. Transplante de Ilhotas de Langerhans.

\footnotetext{
ABSTRACT

Purpose: The long-term effects of five different treatments of diabetes were evaluated in alloxan-induced diabetic rats. Methods: Seven experimental groups, with 50 rats each (GN - normal control; GD - untreated diabetic control; GI, GA, GIA - treated groups with insulin, acarbose, and insulin plus acarbose, respectively; GTIL, GTPD - treated groups with islet of Langerhans and pancreas transplantation) were studied. Clinical
} 
(body weight, water intake, food intake and urine output) and laboratory (blood and urinary glucose, and plasma insulin) parameters were analyzed at the beginning of the study, and after 1, 3, 6, 9 and 12 months of follow-up. Results: Mortality was observed in all groups, except GN, during 12 months (GD=50\%; GI= $20 \%$; GA $=26 \%$; GIA = 18\%; GTIL=4\%; GTPD= 20\%). Rats from the GD, GI, and GIA groups died due to metabolic or hydrossaline disbalance, and/or pneumonia, diarrhoea, and cachexy. All deaths observed in GTIL and GTPD groups were in decorrence of technical failure at the immediate postoperative, until 72h. Animals from the GI, GA and GIA had significative improving of the clinical and laboratory parameters ( $\mathrm{p}<$ 0,05 ) observed in diabetic rats, being the efficacy of theses treatments equal. However, rats from the GTIL and GTPD groups had better control of these parameters than GI, GA, and GIA groups. Transplanted rats had complete restoration, at the normal levels, of all analyzed variables $(\mathrm{p}<0,01)$. Conclusions: Conventional treatments with insulin, acarbose, and insulin plus acarbose improved the severe diabetic state of the alloxandiabetic rats, but pancreas and islet transplantation have a better performance for treatment of diabetes.

Key words: Diabetes. Rats. Alloxan. Insulin. Acarbose. Pancreas Transplantation. Islet of Langerhans Transplantation.

\section{Introdução}

O Diabetes Mellitus é considerado, hoje, um problema de saúde pública mundial, tanto em termos do número de pessoas afetadas, como também, em relação aos custos envolvidos no controle e tratamento de suas complicações ${ }^{1}$.

Projeções para este século indicam que, no ano de 2010, deverão existir cerca de 239 milhões de pessoas diabéticas no mundo ${ }^{2}$, tendo como agravante desta situação um aumento progressivo das taxas de morbidade e mortalidade relacionado à doença, em todo o mundo ${ }^{3}$.

Em que pese os avanços conseguidos no controle da doença, infelizmente o diabetes ainda é uma das principais causas de incapacitação física para o trabalho, tornando o seu portador 25 vezes mais propenso à cegueira, 17 vezes mais susceptível à nefropatia, com chances cinco vezes maiores de amputação de membros e risco duas vezes maior de doença cardiovascular ${ }^{4,5}$.

Diante dessas perspectivas negativas é plenamente justificável a busca de terapêuticas mais eficazes, não só para controlar o metabolismo glicídio, como também, evitar a progressão das lesões crônicas do diabetes sobre os vasos, rins, retina e nervos do paciente diabético $0^{6,7,8}$.

Neste propósito, a reprodução das alterações provocadas pelo diabetes no ser humano, em animais de experimentação, tem sido o caminho encontrado pelos pesquisadores para melhor compreender a fisiopatologia da doença, e também testar o potencial das diversas modalidades terapêuticas no controle das suas alterações clínicas, laboratoriais e histopatológicas.

Assim, este trabalho avaliou, em um estudo experimental de longo prazo, os efeitos de cinco diferentes tratamentos do diabetes, utilizando três terapêuticas convencionais (insulina, acarbose e associação insulina + acarbose) e duas terapêuticas alternativas (transplante de pâncreas e transplantes de ilhotas de Langerhans).

\section{Métodos}

\section{Animais}

O presente estudo foi realizado em ratos Lewis, adultos, machos, alo e isogênicos, com aproximadamente 3 meses de idade e com peso médio de $250 \mathrm{~g}$. Os animais foram fornecidos pelo Biotério Central do Campus da UNESP de Botucatu, tendo sido vermifugados e tratados profilaticamente de pediculose, durante um período de adaptação ao Laboratório Experimental, de 7 dias.

\section{Grupos experimentais}

Sete grupos experimentais, com 50 animais cada um, foram avaliados neste estudo, sendo: GN - grupo controle normal, constituído de ratos sadios nãodiabéticos; GD - grupo controle diabético, constituído de ratos diabéticos, sem qualquer tipo de tratamento; GI - grupo diabético tratado com insulina porcina, monocomponentes (Monotard - Novo NordiskFarm., São Paulo), administrada por via subcutânea, na dose inicial de 18-20 U/kg de peso corporal, ajustada de acordo com a glicosúria, 
realizada duas vezes ao dia; GA - grupo diabético tratado com acarbose (Bayer do Brasil , São Paulo), na dose de 50mg/100g de ração, administrada por via oral; GIA - grupo diabético tratado com insulina associada à acarbose, em doses e vias semelhantes aos grupos GI e GA; GTIL - grupo diabético tratado com transplante de ilhotas de Langerhans, isoladas pelo método da colagenase, e injetadas na veia porta; GTPD - grupo diabético tratado com transplante pancreatoduodenal heterotópico, com drenagem exócrina entérica; cada um dos grupos experimentais foi subdividido em 5 subgrupos, compostos de 10 animais cada, designados de subgrupo $1 \mathrm{M}$ - animais sacrificados com 1 mês de seguimento; subgrupo 3M - animais sacrificados com 3 meses de seguimento; subgrupo 6M - animais sacrificados com 6 meses de seguimento; subgrupo $9 \mathrm{M}$ - animais sacrificados com 9 meses e subgrupo $12 \mathrm{M}$ - animais sacrificados com 12 meses de seguimento.

\section{Indução do diabetes experimental}

O diabetes experimental foi induzida pela administração de uma solução aquosa de aloxana a $2 \%$, injetada por via endovenosa, na dose única de $42 \mathrm{mg} / \mathrm{kg}$ de peso corporal, utilizando umas das veias da cauda do animal.

No presente estudo somente foram utilizados animais com alterações clínicas e laboratoriais compatíveis com diabetes grave, representadas por glicemia de jejum acima de 200mg/dl, glicose urinária maior ou igual $3000 \mathrm{mg} / \mathrm{dl}$, além de perda de peso, polidpsia e poliúria. Os animais que não preencheram esses critérios foram desprezados.

\section{Parâmetros analisados}

Na presente investigação foram avaliados, nos 7 grupos experimentais, os seguintes parâmetros: clínicos (evolução do peso corporal, ingestão hídrica e diurese de 24h e ingestão alimentar de 12h), laboratoriais (dosagem de glicose plasmática, glicose urinária e níveis séricos de insulina). Todos os estudos foram realizados em gaiolas metabólicas no momento M0 (14 ${ }^{\circ}$ dia de seguimento ou de indução do diabetes) e no dia anterior ao sacrifício, após 1, 3, 6, 9 e 12 meses de seguimento.

\section{Isolamento de ilhotas de Langerhans}

As ilhotas de Langerhans foram preparadas com o emprego de colagenase, de acordo com o método padronizado em nosso laboratório ${ }^{9}$, com base no procedimento original descrito por Moskalewski ${ }^{10}$, subseqüentemente modificado ${ }^{11,12}$. Cerca de 15002000 células, oriundas de 4 ratos doadores, foram injetadas na veia porta de cada rato diabético receptor.

\section{Transplante pancreatoduodenal}

O transplante pancreatoduodenal (TPD) foi realizado por microcirurgia segundo a técnica padronizada em nosso meio ${ }^{13}$, com base no procedimento originalmente descrito por Lee e col. ${ }^{14}$.

Para se evitar os efeitos da imunossupressão crônica sobre os resultados, todos os transplantes (pâncreas e ilhotas) foram realizados entre ratos isogênicos. Somente ratos com controle completo do estado diabético foram considerados para análise estatística.

\section{Dosagens laboratoriais}

A glicemia de jejum e a glicose urinária foram dosadas pelo método enzimático (glicose-E, Celm Cia Equipadora de Labs. Modernos Brasil), a pesquisa de glicosúria por fitas reagentes (UriskanGluketo, Bayer Corporation, USA) e a dosagem de insulina por radioimunoensaio, utilizando o método em fase sólida (Coat-A-Coat insulin, DPC, USA).

\section{Análise estatística}

O estudo das variáveis clínicas (peso, ingestão hídrica, ingestão alimentar e diurese) e laboratoriais (glicemia, glicose urinária e insulina), em todos os grupos experimentais, foi realizado pela análise de variância de perfil (7 grupos em 6 momentos), complementado com o teste de comparações múltiplas de Tukey ${ }^{15,16}$ (distribuição simétrica) e análise não paramétrica "Mann-Whitney e KruskalWallis ${ }^{16}$ para as variáveis com distribuição assimétrica. O nível de significância foi definido para $\mathrm{p}<0,05$ ou $5 \%$.

\section{Resultados}

\section{Mortalidade}

Não houve óbitos no grupo normal (GN). Em contraste, a mortalidade foi elevada em ratos submetidos à injeção do aloxana. Quarenta por cento dos ratos morreram até o $14^{\circ}$ dia de pós-indução do diabetes. Outros 20\% não ficaram diabéticos, ou 
apresentaram doença de gravidade leve ou moderada, sendo desprezados. Assim, o índice de obtenção de diabetes grave foi de $40 \%$.

No seguimento clínico, após o $14^{\circ}$ dia, $50 \%$ dos animais do grupo diabético sem tratamento (GD), morreram até o término do experimento, em decorrência de distúrbios metabólicos, pneumonia e/ ou caquexia. Todos os óbitos (pós-indução e no seguimento) foram repostos.

No grupo tratado com insulina (GI), 20\% dos animais morreram no seguimento tardio de 12 meses, devido controle precário da glicemia e/ou pneumonia. Similarmente, $26 \%$ dos ratos tratados com acarbose (GA) morreram até o $12^{\circ}$ mês, em decorrência, principalmente, de distúrbios hidroeletrolíticos causados por diarréia e/ou caquexia. O índice de mortalidade foi menor nos animais tratados com insulina + acarbose (18\%), tendo a diarréia como a principal causa-mortis.

Dois animais (4\%) submetidos ao transplante de ilhotas de Langerhans (GTIL) morreram no pósoperatório imediato, em decorrência de hipertensão portal aguda + trombose portal. Não houve óbitos no seguimento, contudo, 8 animais (17\%) do grupo sobrevivente apresentaram controle parcial do estado diabético.

Vinte por cento dos animais submetidos ao transplante pancreatoduodenal foram à óbito no pósoperatório imediato, até $72 \mathrm{~h}$, em decorrência de falhas técnicas, sendo as principais: trombose da anastomose porto-cava, sangramento pós-reperfusão do enxerto e estenose da anastomose arterial (isquemia do enxerto). Não houve óbitos no seguimento tardio; todos os animais que sobreviveram tiveram controle completo do estado diabético.

\section{Alterações clínicas do diabetes}

Observa-se que ratos sadios do grupo GN apresentaram ganho progressivo de peso corporal, ao longo do estudo, que diferiu significativamente $(\mathrm{p}<0,01)$ do encontrado em ratos diabéticos sem tratamento (GD), onde houve perda progressiva de peso, até a caquexia (Figura 1).

Entre os grupos tratados, observa-se que todas as formas de tratamento convencional, respectivamente, insulina (GI), acarbose (GA) ou associação insulina + acarbose (GIA), não tiveram efeito benéfico significativo $(p>0,10)$ sobre a curva ponderal, quando comparado com ratos diabéticos sem tratamento (GD). Biologicamente, porém, o pior desempenho foi do tratamento com a acarbose utilizada isoladamente.

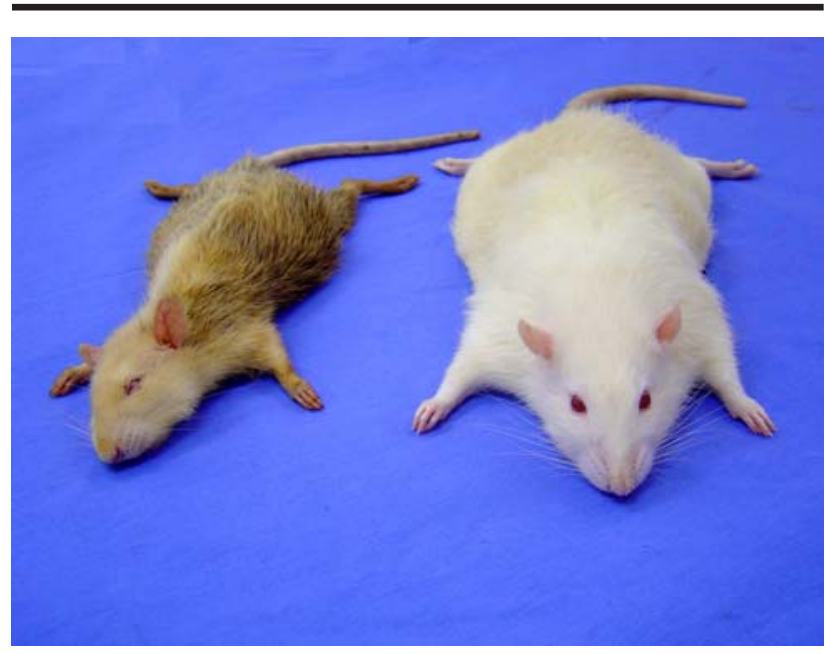

FIGURA 1 - Ilustração de dois ratos, de mesma idade (12m). Note a debilidade, caquexia e alteração da pelagem de um rato diabético sem tratamento (esquerda), quando comparado com o vigor físico de um rato normal

O contrário foi observado com os tratamentos alternativos pelo transplante de ilhotas de Langerhans (GTIL) e transplante pancreatoduodenal (GTPD), bem sucedidos, onde observa-se que a despeito de uma queda inicial do peso corporal, após o trauma cirúrgico, os ratos de ambos os grupos apresentaram uma curva ascendente de peso corporal, que não diferiu estatisticamente da curva ponderal observada em ratos normais sadios (Figura 2).

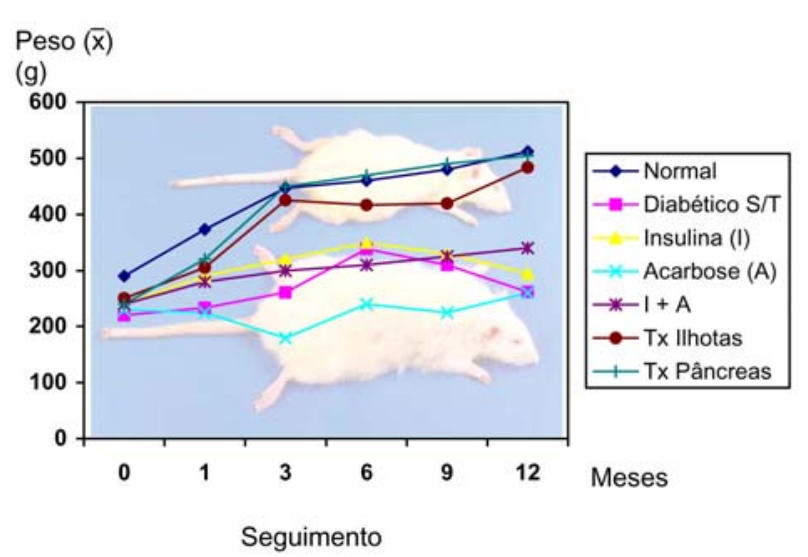

FIGURA 2 - Médias do peso corporal (g) de animais normais, diabéticos não tratados e diabéticos tratados com cinco diferentes tratamentos, durante 12 meses de seguimento 
Em relação à ingestão hídrica observa-se que os volumes ingeridos foram sempre altos em ratos diabéticos sem tratamento (GD), quando comparados com os observados em ratos normais (GN).

Quanto aos tratamentos, observa-se que tanto a insulina, como a acarbose, como a associação de insulina + acarbose determinaram uma redução estatisticamente significativa $(\mathrm{p}<0,05)$ dos valores altos de ingestão hídrica, na maioria dos momentos estudados, quando comparado com ratos diabéticos sem tratamento (GD).

Todavia, os resultados observados em GI, GAe GIA, biologicamente não são comparáveis aos obtidos em ratos diabéticos submetidos ao transplante de ilhotas (GTIL) e de pâncreas (GTPD), onde observou-se correção, aos níveis normais, dos valores altos da ingestão hídrica de 24h, observados em animais do GD, durante todo o seguimento (Figura 3).

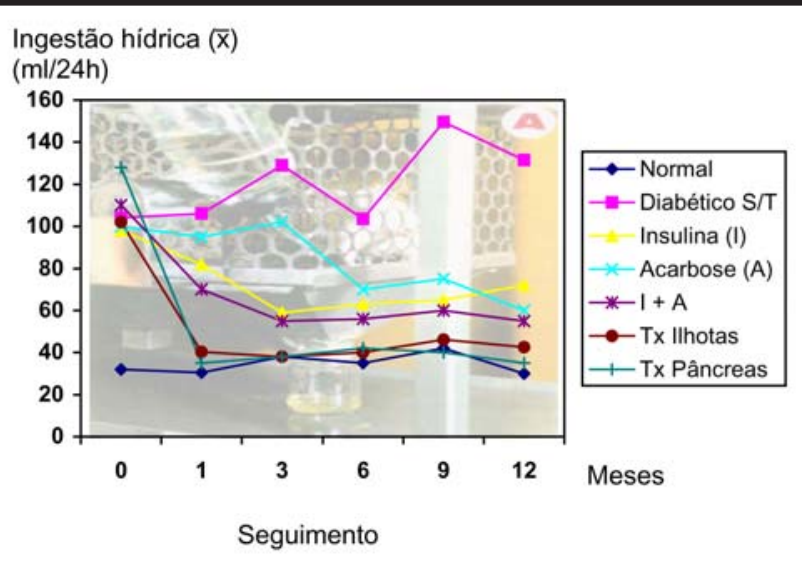

FIGURA 3 - Médias da ingestão hídrica (ml/24h) de animais normais, diabéticos não tratados e diabéticos tratados com cinco diferentes tratamentos, durante 12 meses de seguimento.

A quantidade de alimento ingerido, em $12 \mathrm{~h}$, também foi significativamente maior em ratos do grupo GD, quando comparado com ratos sadios (GN). Porém, este parâmetro mostrou-se ser de difícil avaliação em ratos, em virtude de perdas no recipiente, esfarelamento, retenção de umidade, os quais podem ter afetado a análise fiel dos resultados.

A despeito desses fatores, os animais dos grupos GTIL e GTPD foram os que apresentaram controle total dos valores elevados da ingestão alimentar, durante todo o experimento, quando comparados com outras formas de tratamento. Entre estas, a associação de insulina + acarbose apresentou resultados melhores do que o tratamento com as drogas isoladas (Figura 4).

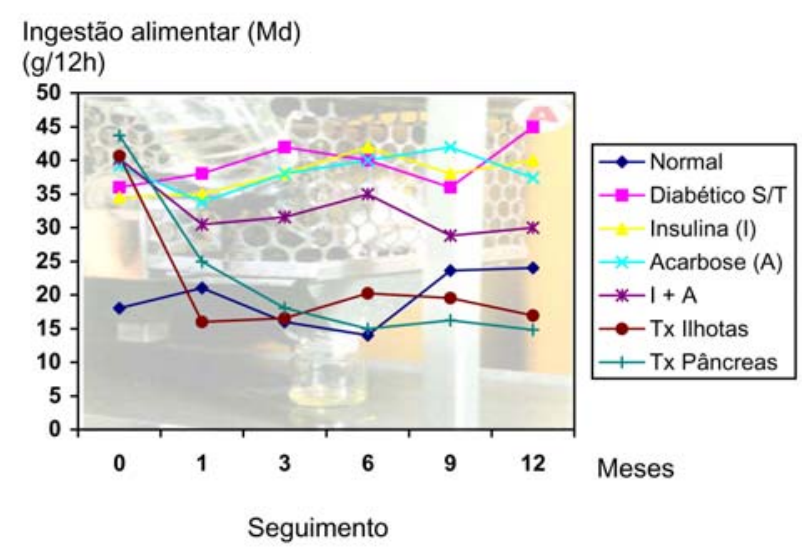

FIGURA 4 - Medianas da ingestão alimentar (g/12h) de animais normais, diabéticos não tratados e diabéticos tratados com cinco diferentes tratamentos, durante 12 meses de seguimento

O comportamento da diurese de $24 \mathrm{~h}$ foi idêntico ao observado com a ingestão hídrica, onde os valores elevados, observados em ratos diabéticos sem tratamento (GD), foram completamente normalizados após o transplante de ilhotas e de pâncreas, bem sucedidos. Novamente, tanto a insulina, como a acarbose, como a associação de insulina + acarbose tiveram efeito positivo sobre o volume de diurese, porém, jamais comparáveis aos transplantes (Figura 5).

\section{Laboratoriais}

Tanto a glicemia de jejum, como a glicose urinária apresentaram valores permanentemente elevados em ratos diabéticos sem tratamento (GD) quando comparados com ratos normais do GN.

Observa-se que todas as modalidades de tratamento apresentaram efeitos benéficos sobre o metabolismo glicídio. Porém, somente o transplante de ilhotas (GTIL) e o transplante de pâncreas (GTPD), bem sucedidos, restabeleceram a glicemia e a glicose urinária, aos níveis normais, durante todo o seguimento. Os demais tratamentos tiveram um comportamento intermediário no controle da glicemia, mas sem diferenças significativas entre eles (Figuras 6 e 7).

A curva de insulina plasmática também foi completamente restabelecida, aos níveis normais, em ratos transplantados. Em ratos do GTPD, inclusive, os valores desse parâmetro ultrapassaram significativamente os valores normais; fato não observado após o transplante de ilhotas de Langerhans. 
Como era de se esperar, os demais tratamentos não interferiram com os valores baixos de insulina, observados em ratos diabéticos (Figura 8).

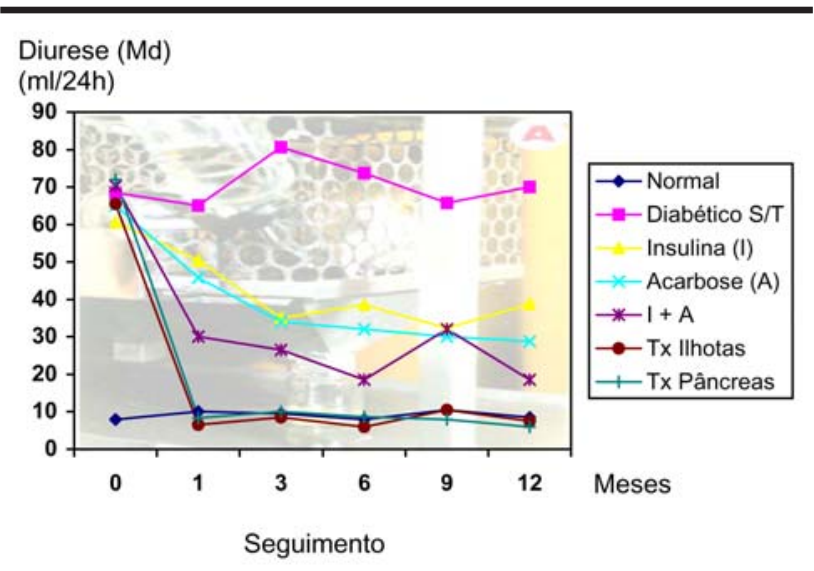

FIGURA 5 - Medianas da diurese (ml/24h) de animais normais, diabéticos não tratados e diabéticos tratados com cinco diferentes tratamentos, durante 12 meses de seguimento

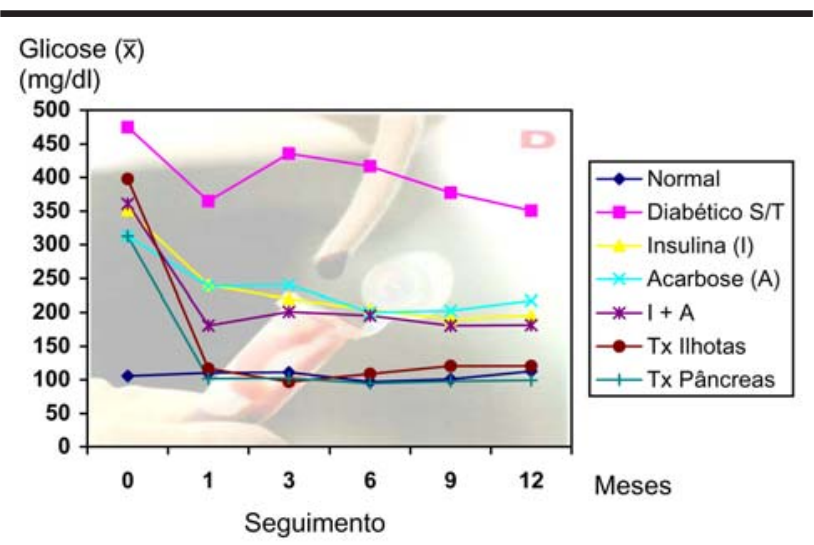

FIGURA 6 - Médias da glicemia (ml/dl) de animais normais, diabéticos não tratados e diabéticos tratados com cinco diferentes tratamentos, durante 12 meses de seguimento

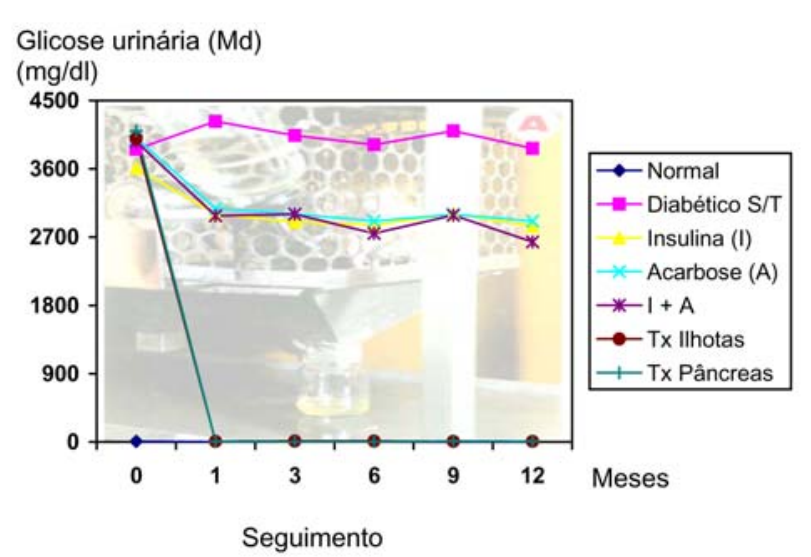

FIGURA 7 - Medianas da glicose urinária (mg/dl) de animais normais, diabéticos não tratados e diabéticos tratados com cinco diferentes tratamentos, durante 12 meses de seguimento

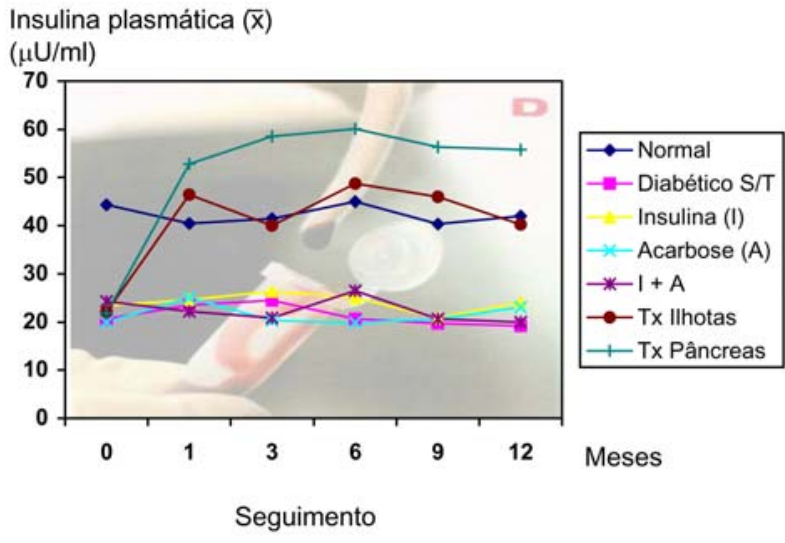

FIGURA 8 - Médias da insulina plasmática (mU/ml) de animais normais, diabéticos não tratados e diabéticos tratados com cinco diferentes tratamentos, durante 12 meses de seguimento

\section{Discussão}

A maioria dos estudos de longo prazo sobre os efeitos das diversas formas de tratamento do Diabetes Mellitus é realizada em modelos-animais. Este tipo de pesquisa tem vantagens sobre os protocolos aplicados em humanos, em razão da possibilidade de se estudar amostras homogêneas, e/ou realizar estudos controlados e randomizados, sem o risco de incorrer em problemas éticos.

Dificilmente seria possível acompanhar o curso de uma moléstia tão grave, como o diabetes, em seres humanos, sem propiciar-lhes alguma forma de tratamento da doença. Isto, no entanto, é possível em animais; com o benefício de possibilitar um período de observação sobre os efeitos da doença, jamais alcançado em humanos.

Considerando a vida média de um rato, em torno de 2 anos, num estudo de 12 meses, como aqui apresentado, isto significaria metade da vida do animal sob os efeitos danosos do diabetes; o que extrapolado para um homem, com expectativa de vida de 70 anos, isto representaria 35 anos de diabetes.

Em nosso estudo demonstramos que qualquer que seja o tratamento instituído para tratar o diabetes insulino-dependente, tratar é sempre melhor do que não tratar o diabetes, haja vista que por pior que tenha sido o controle metabólico obtido com o tratamento, as taxas de morbidade e mortalidade diminuíram significativamente, quando comparadas com as observadas em animais sem tratamento.

Infelizmente, porém, os resultados obtidos com 
os tratamentos convencionais do diabetes, utilizando insulina, acarbose ou a associação de insulina + acarbose, em geral, propiciaram um controle insuficiente e/ou precário do metabolismo glicídio; o que particularmente foi muito ruim, haja vista que a maioria dos pacientes diabéticos no mundo utiliza estas formas de tratamento do diabetes.

A ineficácia da insulinoterapia em controlar adequadamente o metabolismo diabético, e por conseqüência, os efeitos danosos da doença sobre os vasos, rins, retina e nervos do paciente, a longo prazo, tem sido descrita por vários autores na literatura ${ }^{17-21}$. Isto ocorre porque a administração exógena de insulina não consegue manter níveis fisiológicos de hormônio circulante durante as 24h do dia. Segundo Molnar, Taylor ${ }^{22}$ os níveis após a administração de insulina são muito elevados no jejum, os pós-prandiais excessivamente baixos, e o esperado pico pós-prandial de insulina na veia porta não ocorre, prejudicando assim o papel do fígado na metabolização da carga oral de glicose. Não obstante estas observações, diversas evidências clínicas e experimentais, incluindo o uso de bombas de insulina, têm mostrado que um controle mais adequado da glicose sanguínea é possível, com redução das complicações do diabetes sobre o metabolismo e as lesões crônicas ${ }^{23-26}$.

A acarbose, um complexo oligossacarídeo inibidor competitivo da alfa-glicosidase intestinal, é capaz de causar má absorção dos carboidratos da dieta, sem interferir com a absorção de glicose ${ }^{27}$. Esta droga foi introduzida na terapêutica do Diabetes Mellitus com potencialidades para manter níveis mais uniformes de glicose circulante, tanto em pacientes $^{28,29}$, como em animais ${ }^{30}$, utilizada isoladamente ou em associação com a insulina.

Todavia, no estudo aqui apresentado, o tratamento de animais diabéticos com acarbose ou associação de insulina + acarbose, se associou a índices de complicações e de mortalidade que não diferiram dos observados em animais tratados exclusivamente com insulina. A diarréia aquosa, com perdas hidroeletrolíticas importantes, associada à perda de peso, foi a principal complicação observada com o uso desta substância.

Apesar deste aspecto negativo, a administração de acarbose isolada, ou associada, demonstrou ser capaz de reduzir os níveis elevados da ingestão hídrica, ingestão alimentar e diurese, bem com os níveis elevados de glicose sanguínea e urinária de ratos diabéticos; muito embora, estes benefícios não diferiram estatisticamente daqueles observados com o tratamento exclusivo com insulina.

Paradoxalmente, porém, estudo anterior realizado no nosso laboratório demonstrou que o tratamento do diabetes com a associação de insulina + acarbose, ainda que incapaz de propiciar um controle estrito do metabolismo glicídio, tem potencial para prevenir o espessamento da membrana basal e a expansão mesangial de glomérulos de ratos diabéticos aloxânicos, acompanhados por 12 meses $^{31}$, achados estes também observados em outros trabalhos experimentais, mesmo com o uso da acarbose isolada ${ }^{32,33}$.

Essas observações sugerem que, possivelmente, a acarbose tem uma ação mais eficaz que a insulina isolada,na manutenção dos níveis de glicose circulantes, durante as $24 \mathrm{~h}$ do dia.

Em que pese a boa perspectiva do uso da acarbose em associação com a insulina, no tratamento do diabetes, nosso estudo demonstrou que nenhum dos tratamentos convencionais testados foi mais eficaz, no controle do metabolismo diabético, do que o tratamento através dos transplantes de pâncreas e de ilhotas de Langerhans (GTPD e GTIL).

Esses procedimentos, quando bem sucedidos, foram capazes de restaurar completamente o estado euglicêmico, e todas as alterações clínicas observadas no rato diabético, já a partir do pós-operatório imediato. Estas observações são compatíveis com as relatadas por diversos pesquisadores em todo o mundo, tanto em trabalhos clínicos, como experimentais $^{34-37}$.

Todavia, a opção pelo tratamento do diabetes, através dos transplantes de pâncreas e de ilhotas de Langerhans, deve levar em conta os riscos e complicações advindas dos procedimentos terapêuticos, haja vista os índices de falhas técnicas observadas, respectivamente, de 20 e 4\%.

Extrapolados esses dados à situação clínica, os riscos desses procedimentos ainda devem ser somados aos riscos da imunossupressão crônica, a qual não foi utilizada neste experimento.

\section{Conclusões}

A aloxana administrada por via endovenosa produz diabetes franco em ratos, caracterizada por 
alterações clínicas e laboratoriais graves.

Essas alterações podem ser melhoradas com os tratamentos convencionais utilizando insulina, acarbose ou associação de insulina + acarbose; contudo, jamais em níveis compatíveis com a normalidade, como observado com os tratamentos através dos transplantes de pâncreas e de ilhotas de Langerhans.

Embora os benefícios advindos dessas terapêuticas devam ser confrontados com os eventuais riscos dos procedimentos, não há dúvidas de que os transplantes de pâncreas e de ilhotas de Langerhans têm grande potencial para prevenir, estabilizar ou mesmo reverter as lesões crônicas do diabetes.

\section{Referências}

1. Diabetes Complications and Control Trial (DCCT). Research Group. The effect of long-term complications of insulin-dependent diabetes mellitus. N Engl J Med. 1993;329:977-86.

2. Sociedade Brasileira de Diabetes. Diabetes: Consenso da Sociedade Brasileira do Diabetes - CSBD, 2002 (Online), 2004. [acesso 2004 jan 15]. Disponível em: http:// diabetes.org.br/diabetes/info_medicos/consenso/ cons set.html.

3. Malerbi DA, Franco LJ. Multicenter study of the prevalence of diabetes mellitus and impaired glucose tolerance in the urban brazilian population aged 30-69yr. Diabetes Care. 1992;15:1509-16.

4. La Porte RE. Criuckshanks: incidence and risk factors for insulin-dependent diabetes. In: Harris MI, Hamman RF, editors. Diabetes in America. Bethesda: Nacional Diabetes Data Group, NIADDK/US, Dept Health and Human Services; 1985. p. 1-12. (NIH publ. no 85-1468).

5. Królewski AS, Warram JH, Rand LI, Kann CR. Epidemiologic approach to the etiology of type I diabetes mellitus and its complications. $\mathrm{N}$ Engl J Med. 1987;317:1390-8.

6. Pozza G, Secchi A, Bosi E, Pontiroli AE, Micossi P, Spotti D, Platti PM, Gelet A, Touraine JL, Dubernard JM, Traeger J. Endocrine and metabolic profiles in insulindependent diabetics treated with continuous subcutaneous insulin infusion and pancreas transplantation: a comparison. Transplant Proc. 1984;16:1274-6.

7. Ryan EA, Lakey RT, Rajotte RV, Korbutt GS, Kin T, Imes S, Rabinovitch A, Elliot JF, Bigam D, Kneteman NM, Warnock GL, Larsen I, Shapiro AMJ. Clinical outcomes and insulin secretion after islet transplantation with the Edmonton protocol. Diabetes. 2001;50:710-9.

8. Gruessner AC, Sutherhand DER. Analyses of pancreas transplant outcomes for United States cases reported to the United Network for Organ Sharing (UNOS) and nonUS cases reported to the International Pancreas Transplant
Registry (IPTR). Clin Transplant. 1999;23:51-69.

9. Spadella CT, Breim LC, Mercadante MCS, Macedo CS. Transplante de ilhotas de Langerhans: estudo comparativo entre dois métodos diferentes de isolamento de ilhotas do pâncreas do rato. Arq Gastroenterol. 1997;34:27-33.

10. Moskalewski S. Isolation and culture of the islets of Langerhans of the guinea pig. Gen Comp Endocrinol. 1965;5:342-53.

11. Lacy PE, Kostianovsky: method for the isolation of intact islet of Langerhans from the rat pancreas. Diabetes. 1967;16:35-9.

12. Sorenson RL, Lindall AW, Lazarow A. Studies on the isolated goosefish insulin secretion granule. Diabetes. 1969;18(3):129-37.

13. Spadella CT, Mercadante MCS, Machado JLM, Schellini SA, Macedo AR. Transplante pancreato-duodenal no rato com microcirurgia: a técnica e os resultados após 12 anos de experimentação. Arq Gastroenterol. 1996;33:158-66.

14. LEE S, Tung KSK, Koopman H, Chandler JE, Orloff MJ. Pancreaticoduodenal transplantation in the rat. Transplantation. 1972;13:421-5.

15. Montgomery DC. Design and analyses of experiments. 3ed. New York: John Wiley; 1991.

16. Fisher LD, Belle GV. Biostatistcs metodology for the health sciences. New York: Wiley-Inter Science; 1993.

17. Cahill GF Jr, Etzwiller DD, Freinkel N. Control and diabetes. N Engl J Med. 1976;294:1004-5.

18. Knatterud GL, Klimt CR, Levin ME, Jacobson ME, Goldner MG. Effects of hypoglycemic agents on vascular complications in patients with adult-onset. VII. Mortality and selected nonfatal events with insulin treatment. J Am Med Assoc. 1978;240:37-42.

19. Tchobroutsky G. Relation of diabetic control to development of microvascular complications. Diabetologia. 1978;15:143-52.

20. Kolata GB. Controversy over study of diabetes drugs continues for nearly a decade. Science. 1979;230:986-90.

21. Siperstein MD. Diabetic microangiopathy and the control of blood glucose. N Engl J Med. 1983;309:1577-9.

22. Molnar GD, Taylor WF. Day-to-day variation of continuously monitored glycaemia: a further measure of diabetes instability. Diabetologia. 1972;8:342-8.

23. Unger RH. Meticulous control of diabetes: benefits, risks, and precautions. Diabetes. 1982;31:479-83.

24. Tamborlane WV, Sherwin RS, Genet M, Felig P. Restoration of normal lipid and amino acid metabolism in diabetic patients treated with a portable insulin infusion pump. Lancet. 1979;1(8129):1258-61.

25. Viberti GC, Pickup JC, Jarret T, Keen H. Effect of control of blood glucose on urinary excretion of albumin and bmicroglobulin in insulin-dependent diabetes. N Engl J Med. 1979;300(12):638-61.

26. Raskin P, Pietri AO, Unger R, Shannon WA Jr. The effect of diabetic control on the width of skeletal-muscle capillary basement membrane in patients with type I diabetes mellitus. N Engl J Med. 1983;309(25):1546-60.

27. Caspary WF. Sucrose malabsorption in man after 
ingestion of alpha-glucosidehydrolase inhibitor. Lancet. 1978;1:1231-3.

28. Sachse G, Willms B. Effect of the alpha-glycosidase inhibitor Bay-g-5421 on blood glucose control of sulphonylurea treated diabetics and insulin treated diabetics. Diabetologia. 1979;17:287-90.

29. Hoffman J, Spengler M. Efficacy of 24 week monotherapy with acarbose, glibenclamide or placebo in NIDDM patients. Diabetes Care. 1994;17:561-6.

30. Katovich MJ, Meldrum MJ, Vasselli JR. Beneficial effects in the streptozotocin-induced diabetic rat. Metabolism. 1991;40:1275-82.

31. Macedo CS, Silva MD, Spadella CT, Breim LC, Capeletti SM, Mercadante MCS, Hernandes D, Macedo AR. Effect of long-term treatment with insulin and/or acarbose on glomerular basement membrane thickening in alloxandiabetic rats. Braz J Med Biol Res. 1996;29:1329-35.

32. Chakrabarti S, Cherian PV, Simas AA. The effect of acarbose on diabetes and age-related basement membrane thickening in retinal capillaries on the BB/W rat. Diabetes Res Clin Pract. 1993;20:123-8.

33. Sima AA, Chakrabarti S. Long-term suppression of postprandial hyperglycemia with acarbose retards the development of neuropathies in the $\mathrm{BB} / \mathrm{W}$ rat. Diabetologia. 1992;34:325-30.
34. Östman J, Bolinder J, Gunnarson R, Brattström C, Tydén G, Wahren J, Groth C. Effects of pancreas transplantation on metabolic and hormonal profiles in IDDM patients. Diabetes. 1989;38(suppl 1):88-93.

35. Orloff MJ, Macedo CS, Macedo AR, Greenleaf GE. Comparison of whole pancreas and pancreatic islet transplantation in controlling nephropathy and metabolic disorders of diabetes. Ann Surg. 1987;206:324-34.

36. Spadella CT, Schellini SA, Bacchi CE. Pancreas transplantation versus islet transplantation versus insulin therapy in the prevention of nephropathy in alloxan-induced diabetic rats. Transplant Proc. 1998;30:327-9.

37. International Pancreas Transplant Registry (IPTR). Transplant pancreas: University of Minnesota (USA), 2003 [Online], 2004. [acesso 2004 jan. 15]. Disponível em: http://www.iptr.umn.Edu/ar_midyear_update_index.htm.

\section{Agradecimentos}

Os autores agradecem as Sras. Maria Cecília Salgado Mercadante, Sônia Maria Capeletti e Irene Spago pelo auxílio na execução dos procedimentos técnicos e no seguimento clínico dos animais.
Correspondência:

Prof. Adj. César Tadeu Spadella

Departamento de Cirurgia e Ortopedia, Faculdade de Medicina

Campus de Botucatu, UNESP

18618-970
Conflito de interesse: nenhum Fonte de financiamento: Fapesp

Recebimento: 06/10/2004 Revisão: 28/11/2004

Aprovação: 29/12/2004

\section{Como citar este artigo:}

Spadella CT, Macedo CS, Machado JLM, Schellini SA, Padovanni CR. Estudo comparativo entre cinco diferentes tratamentos sobre as alterações clínicas e laboratoriais do rato diabético induzido pela aloxana. Acta Cir Bras. [serial online] 2005 JanFev;20(1). Disponível em URL: http://www.scielo.br

*Figuras coloridas disponíveis em $\underline{w w w . s c i e l o . b r / a c b}$ 